\title{
A spin crossover ferrous complex with ordered magnetic ferric anions
}

\author{
Olivier Roubeau, ${ }^{a}{ }^{a}$ Eva Natividad, ${ }^{b}$ Marco Evangelisti ${ }^{a}$ \\ Received (in $X X X, X X X) X$ th $X X X X X X X X X 20 X X$, Accepted $X$ th $X X X X X X X X X 20 X X$ \\ DOI: 10.1039/c0xx00000x
}

\begin{abstract}
${ }_{5}$ The first tetrahaloferrate spin crossover compound, $\left[\mathrm{Fe}(\mathrm{Metz})_{6}\right]\left(\mathrm{FeBr}_{4}\right)_{2}$, is reported. The $\mathrm{FeBr}_{4}^{-}$ions form ferromagnetically coupled 1D stacks and exhibit an antiferromagnetic order at $2.2 \mathrm{~K}$, that coexists with the gradual spin crossover centred at $165 \mathrm{~K}$.
\end{abstract}

10 Designing magnetic materials with two or more potential functions resulting from different physical properties, either in synergy or totally un-correlated, has become a strong topic in chemical and materials sciences. ${ }^{1}$ Spin crossover (SCO) compounds have been the source of a number of multifunctional 15 synthetic systems, ${ }^{2}$ as the SCO phenomenon itself, in particular with $\mathrm{Fe}(\mathrm{II})$ coordination systems for which the low-spin (LS) state is purely diamagnetic, gives access to concomitant drastic variations in magnetic, optical and dielectric responses, ${ }^{3}$ and can be implemented into liquid crystalline or gel phases. ${ }^{4}$ An elegant 20 synthetic strategy towards multifunctional materials is to build two-network hybrid crystalline solids, and this has been particularly efficient with one of the networks having a 2D layered structure thus allowing intercalation of molecular, $1 \mathrm{D}$ or 2D species. ${ }^{5}$ SCO complexes were only recently successfully 25 integrated into $2 \mathrm{D}$ and $3 \mathrm{D}$ ferromagnetic magnetic oxalate anionic networks, ${ }^{6}$ using mononuclear Fe(III) complexes bearing chelating ligands. Fe(II) systems have so far eluded such success, likely because of a combination of inadequate charge, easier oxidation and greater sensitivity to environmental changes of the 30 conditions favouring SCO. On the other hand, Fe(II) SCO complexes were successfully integrated into materials with lower dimensionality, as cations ${ }^{7}$ or spin component ${ }^{8}$ of Single-ChainMagnets, although in the latter case SCO was not maintained. With the goal of obtaining simpler truly molecular SCO 35 compound with interesting magnetic properties at low temperatures, we have used paramagnetic tetrabromoroferrate(III) anions in conjunction with 1-methyltetrazole (Metz). Indeed, the $\left[\mathrm{Fe}^{\mathrm{II}}\left(\mathrm{N}_{\mathrm{tz}}\right)_{6}\right]$ coordination core ( $\mathrm{tz}=$ substituted tetrazole) probably represents the most-widely studied type of SCO compounds, 40 while providing the highest probability of occurrence of SCO. ${ }^{9}$ On the other hand, tetrahaloferrate(III) ions have been widely used in molecular conductors ${ }^{10}$ and can interact ferromagnetically depending on their stacking, ${ }^{11}$ potentially resulting in ferromagnetic order. ${ }^{12}$ We report here the first SCO compound 45 with a tetrahaloferrate(III) anion, $\left[\mathrm{Fe}(\mathrm{Metz})_{6}\right]\left(\mathrm{FeBr}_{4}\right)_{2}$ (1) and its analogue $\left[\mathrm{Zn}(\mathrm{Metz})_{6}\right]\left(\mathrm{FeBr}_{4}\right)_{2}$ (2), in which the $\mathrm{FeBr}_{4}{ }^{-}$ions form ferromagnetically coupled $1 \mathrm{D}$ stacks and order antiferromagnetically at $2.2 \mathrm{~K}$.

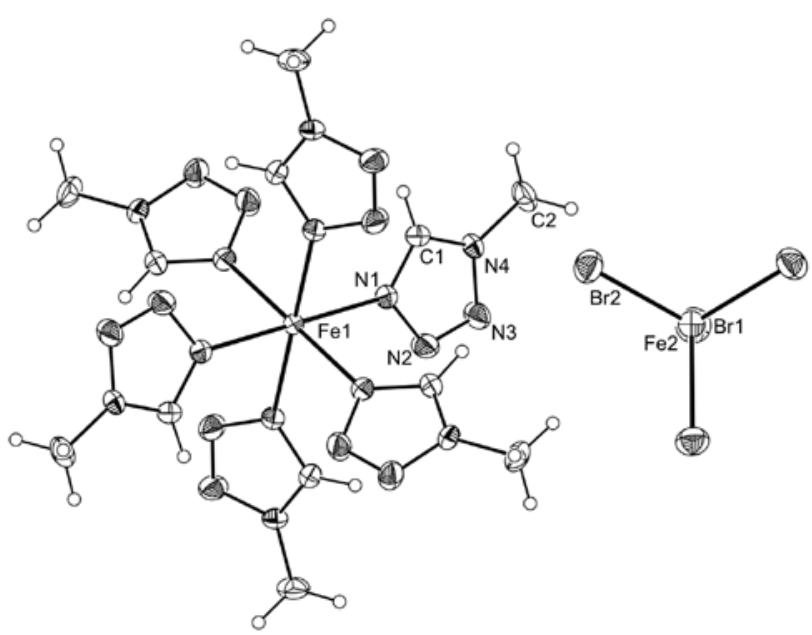

50 Fig. 1 ORTEP view at $30 \%$ probability of the structure of 1 at $296 \mathrm{~K}$ along the $c$ axis. Only non-hydrogen atoms of the asymmetric unit are labelled.

Red crystals of $\mathbf{1}$ are readily obtained in high yield from the reaction of Metz, ferrous and ferric bromides in a 6:1:2 molar 55 ratio in ethanol. The $\mathrm{Zn}(\mathrm{II})$ analogue, $\left[\mathrm{Zn}(\mathrm{Metz})_{6}\right]\left(\mathrm{FeBr}_{4}\right)_{2}(2)$ is easily obtained replacing ferrous bromide by zinc bromide. This simple synthetic route uses $\mathrm{FeBr}_{4}{ }^{-}$ions formed in-situ from $\mathrm{FeBr}_{3}$ in the presence of bromide anions, thus avoiding the addition of a pre-formed salt that would add an unwanted cation to the system. 60 In addition, the presence of Fe(III) ions in solution likely prevents $\mathrm{Fe}(\mathrm{II})$ ions from aerobic oxidation, as no addition of ascorbic acid is required for the synthesis of $\mathbf{1}$.

Single-crystal X-ray analysis at room temperature reveals $\mathbf{1}$ crystallizes in the trigonal $P-3$ space group with one ${ }_{65}\left[\mathrm{Fe}(\mathrm{Metz})_{6}\right]^{2+}$ cation and two $\mathrm{FeBr}_{4}^{-}$ions per cell. The asymmetric unit contains two Fe sites on special positions with 6 and 3-fold multiplicity respectively, one Metz molecule and two $\mathrm{Br}$ atoms. The $\left[\mathrm{Fe}(\mathrm{Metz})_{6}\right]^{2+}$ cation consists of Fe1 atom surrounded by six Metz ligands, identical by symmetry, 70 coordinated through N1 with a Fe-N1 bond distance of 2.186(2) $\AA$, indicative of a HS state. ${ }^{13}$ The resulting $\mathrm{FeN}_{6}$ octahedron is only slightly distorted with $\mathrm{N}-\mathrm{Fe}-\mathrm{N}$ angles of 88.88(9) and 91.12(9) ${ }^{\circ}$. Each methyl group points towards another from a neighbouring complex, with a C2 $\cdots \mathrm{C} 2$ ' separation of $3.354 \AA$. ${ }_{75} \mathrm{The}_{\mathrm{FeBr}}{ }_{4}^{-}$ion has an almost perfect tetrahedral geometry around the $\mathrm{Fe} 2$ atom with similar $\mathrm{Fe} 2-\mathrm{Br} 1$ and $\mathrm{Fe} 2-\mathrm{Br} 2$ bond distances, at respectively 2.3240(11) and 2.3437(5) $\AA$, and Br1-Fe-Br2 and $\mathrm{Br} 2-\mathrm{Fe}-\mathrm{Br} 2$ bond angles, at respectively $109.70(2)$ and 
$109.24(2)^{\circ}$, in accord with simple salts of this anion. ${ }^{14}$ Each $\mathrm{FeBr}_{4}^{-}$ion forms hydrogen bonds with a tetrazole aromatic hydrogen of three neighbouring $\left[\mathrm{Fe}(\mathrm{Metz})_{6}\right]^{2+}$ complexes, with a $\mathrm{Br} 2 \cdots \mathrm{H} 1$ distance of 3.811(4) $\AA$ (Fig. S2). The two $\mathrm{FeBr}_{4}{ }^{-}$in the 5 cell have their Fe2-Br1 bond parallel to the $c$ axis, in opposite directions, with a $\mathrm{Fe}^{\cdots} \cdot \mathrm{Fe}$ separation of $7.489 \AA$ and a $\mathrm{Br} 2 \cdots \mathrm{Br} 2$ short contact of $4.472 \AA$ (Fig. S3). The $\mathrm{FeBr}_{4}{ }^{-}$ions are stacked along the $c$ axis in a perfectly eclipsed conformation (Fig. S3), with a Fe $\cdots$ Fe separation of 6.9577(1) $\AA$. There are rather short 10 $\mathrm{Br} 1 \cdots \mathrm{Br} 2$ and $\mathrm{Br} 1 \cdots \mathrm{Fe} 2$ contacts between these stacked $\mathrm{FeBr}_{4}{ }^{-}$at respectively 4.431 and $4.634 \AA$ (Figs. S3 and S4). A similar structure is obtained at $104 \mathrm{~K}$, with no change in space group but with a reduction of both $a$ and $c$ axes, resulting in a cell volume decrease of $6.2 \%$. This reduction likely involves both the normal 15 thermal contraction and the effect of the SCO occurring at the Fe1 site. Indeed, the Fe1-N1 bond distance has decreased by $8.4 \%$ at $2.003(2) \AA$, now typical of a LS state. ${ }^{13}$ The Fe $\cdots$ Fe separation along the $\mathrm{FeBr}_{4}^{-}$stacks is reduced, to 6.7980(14) $\AA$, with shorter $\mathrm{Br} 1 \cdots \mathrm{Br} 2$ and $\mathrm{Br} 1 \cdots \mathrm{Fe} 2$ contacts, at respectively 204.287 and $4.474 \AA$. The inter-stack Fe $\cdots$ Fe separation is also reduced, to $7.337 \AA$. The $\mathrm{Zn}(\mathrm{II})$ analogue $\mathbf{2}$ is isostructural to $\mathbf{1}$ at room temperature, with very similar stacking of the $\mathrm{FeBr}_{4}{ }^{-}$ions (see Table S1 and Fig. S5).

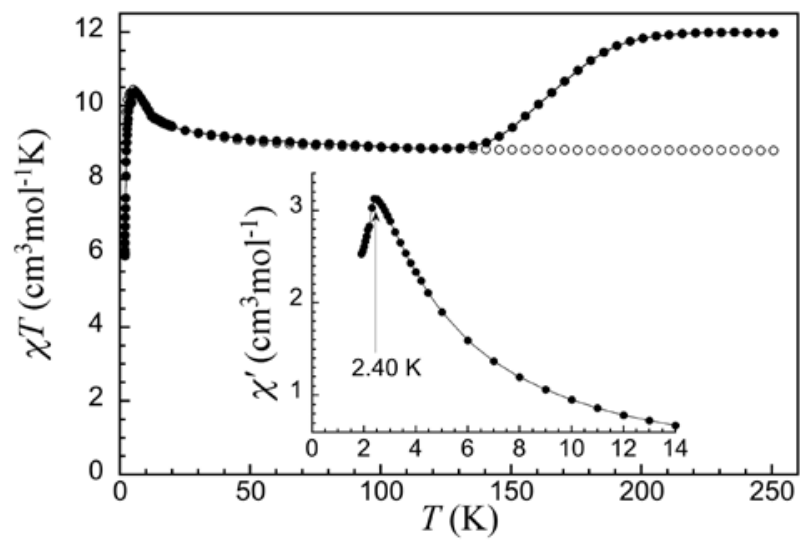

${ }_{25}$ Fig. $2 \chi T$ vs. $T$ plot for compounds 1 (full circles) and 2 (empty circles) showing the process of SCO in $\mathbf{1}$ and a similar ferromagnetic signature down to $6 \mathrm{~K}$ for both $\mathbf{1}$ and 2 . Inset: low-temperature in-phase as susceptibility of $\mathbf{1}$ at $15 \mathrm{~Hz}$.

Magnetic properties of both compounds were determined in 30 the range $1.9-250 \mathrm{~K}$ and are sketched in Figure 2 as a $\chi T$ vs. $T$ plot, $\chi$ being the molar magnetic susceptibility. The value of $\chi T$ at $250 \mathrm{~K}$ for compound $\mathbf{1}$, at $12.0 \mathrm{~cm}^{3} \mathrm{~mol}^{-1} \mathrm{~K}$, agrees with that expected for one $\mathrm{Fe}(\mathrm{II})$ ion in the $S=2 \mathrm{HS}$ state and two $\mathrm{Fe}(\mathrm{III}) S$ $=5 / 2$ spins $\left(11.75 \mathrm{~cm}^{3} \mathrm{~mol}^{-1} \mathrm{~K}\right)$. A decrease of $\chi T$ is observed 35 upon cooling from about $210 \mathrm{~K}$ reaching a minimum at around $120 \mathrm{~K}$ at $8.84 \mathrm{~cm}^{3} \mathrm{~mol}^{-1} \mathrm{~K}$, value in accord with only two Fe(III) $S$ $=5 / 2$ spins $\left(8.75 \mathrm{~cm}^{3} \mathrm{~mol}^{-1} \mathrm{~K}\right)$. Clearly this decrease corresponds to the almost complete thermal SCO exhibited by the Fe1 site, and centred at ca. $170 \mathrm{~K}$. Indeed, compound 2, in which this site 40 is occupied by a diamagnetic $\mathrm{Zn}(\mathrm{II})$ ion, exhibits similar $\chi T$ values, at $8.79-8.86 \mathrm{~cm}^{3} \mathrm{~mol}^{-1} \mathrm{~K}$, from 250 down to about $115 \mathrm{~K}$. The gradual SCO behaviour of $\mathbf{1}$ is similar in shape to that of the $\mathrm{BF}_{4}^{-}$or $\mathrm{ClO}_{4}^{-}$derivatives, but it occurs ca. $100 \mathrm{~K}$ higher in temperature. $^{9}$ Moreover, the presence of a single $\mathrm{Fe}(\mathrm{II})$ 45 crystallographic site in $\mathbf{1}$ results in a complete SCO, as opposed to the $50 \%$ SCO associated with the presence of two Fe sites of these other derivatives with tetrahedral anions. ${ }^{9,14}$ Below $120 \mathrm{~K}$, both compounds $\mathbf{1}$ and $\mathbf{2}$ exhibit a similar increase of $\chi T$, indicative of the presence of ferromagnetic interactions, to reach 50 a maximum value of $10.38 \mathrm{~cm}^{3} \mathrm{~mol}^{-1} \mathrm{~K}$ at $6 \mathrm{~K}$. Below $6 \mathrm{~K}$, a sharp decrease is then observed, to reach $6.09 \mathrm{~cm}^{3} \mathrm{~mol}^{-1} \mathrm{~K}$ at $1.9 \mathrm{~K}$ for $\mathbf{1}$. The increase of $\chi T$ can reasonably be ascribed to a ferromagnetic coupling of the paramagnetic $\mathrm{FeBr}_{4}{ }^{-}$ions in the stacked chains along the $c$ axis. ${ }^{11}$ The drop below $6 \mathrm{~K}$, associated with a marked 55 maximum at $2.40 \mathrm{~K}$ in $\chi$ ' (inset in Fig. 2) likely corresponds to an antiferromagnetic order of the $\mathrm{FeBr}_{4}{ }^{-}$ions.

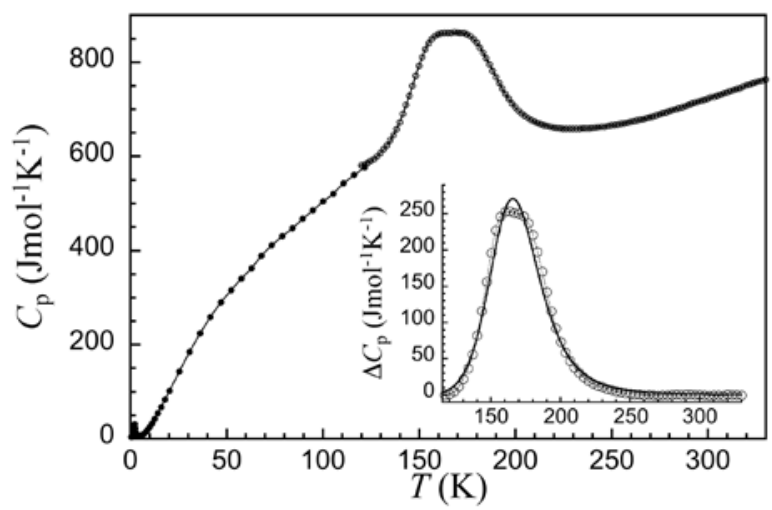

Fig. 3 The molar heat capacities of $\mathbf{1}$ showing a broad hump associated with the SCO. Full/empty symbols: relaxation/DSC data. Inset: Excess 60 heat capacities associated with the SCO in $\mathbf{1}$. The full line is a fit to the domain model of Sorai (see text and SI).

The molar heat capacity $\left(C_{\mathrm{p}}\right)$ of $\mathbf{1}$ was determined from differential scanning and semi-adiabatic relaxation calorimetry for the temperature ranges $120-330 \mathrm{~K}$ and $0.3-130 \mathrm{~K}$, 65 respectively. We associate the broad hump covering 130-220 K (Fig. 3) to the thermal signature of a gradual SCO, in good agreement with the magnetic data. The excess enthalpy and entropy associated with the SCO in $\mathbf{1}$ were deduced by integration of the excess heat capacity (inset in Fig. 3) and arise 70 to $13.1 \mathrm{kJmol}^{-1}$ and $77.8 \mathrm{Jmol}^{-1} \mathrm{~K}$, respectively. These figures are rather large, in particular the excess entropy well above the electronic component $R \ln 5$, which is often taken as an indication of the cooperativeness of the SCO phenomenon. Nevertheless, reproducing the experimental data with the so-called domain 75 model $^{15}$ gives a domain size $n$ of 1.47 , in agreement with a poorly-cooperative system ${ }^{15,16}$ and thus with the shape of magnetic and thermal signature of the SCO in $\mathbf{1 .}$

The low-temperature heat capacity is characterized by an applied-field dependent contribution, whose most prominent 80 feature is a lambda-like peak centred at $T_{N} \cong 2.2 \mathrm{~K}$ for zero-field (Fig. 4). This feature indicates a phase transition to long-range magnetic order, nicely corroborating the magnetic data. The fact that the peak is not affected by fields lower than $B_{0}=0.5 \mathrm{~T}$ points to antiferromagnetic ordering. Larger fields gradually decouple 85 the magnetic interactions and for, e.g. $B_{0}=7 \mathrm{~T}$ in Fig. 4, the experimental $C_{\mathrm{p}}$ is satisfactorily modelled with the Schottky heat capacity (solid line), which results from summing the contributions of two non-interacting Fe(III) ion spins. At high temperatures, the experimental $C_{\mathrm{p}}$ is dominated by non-magnetic 90 contributions arising from thermal vibrations of the lattice, which 
can be modelled with the Debye function (dashed line) yielding a value of $\theta_{\mathrm{D}}=43.4 \mathrm{~K}$ for the Debye temperature, which is in the range of values observed for this class of molecular compounds. ${ }^{17}$ By subtracting the lattice contribution from the zero-field $C_{\mathrm{p}}$, we 5 are left with the tail of the magnetic ordering heat capacity $\left(C_{\mathrm{m}}\right)$ that has a characteristic $T^{-2}$ dependence at high temperatures (empty markers for $T>3 \mathrm{~K}$ ). On the other side of the peak, we note an upward curvature below $0.5 \mathrm{~K}$, which can be understood as an excess $C_{\mathrm{p}}$ added on top of the zero-field peak data. As 10 indicated in Fig. 4 (empty markers for $T<0.5 \mathrm{~K}$ ), we have used a linear- $T$ extrapolation, as would be appropriate for the spin-wave contribution for an isotropic 1D antiferromagnet. ${ }^{18}$ The resulting low values for the excess $C_{\mathrm{p}}$, combined with its slight dependence on the applied field, suggests that this feature originates from the 15 splitting of residual HS Fe(II) ions by the internal ordering field. Finally, associating the spin-wave extrapolation to $C_{\mathrm{m}}$, we obtain the zero-field magnetic entropy, using $S_{m}(T)=\int_{C_{m}}(T) / T \mathrm{~d} T$. The inset of Fig. 4 shows that $S_{\mathrm{m}}(T)$ beautifully tends at high $T$ to the full content corresponding to two $\mathrm{Fe}(\mathrm{III}) S=5 / 2$ spins, i.e. $202 x R \ln (2 S+1)=3.58 R$, corroborating our initial hypothesis that the ordering involves $\mathrm{Fe}(\mathrm{III})$ spins solely.

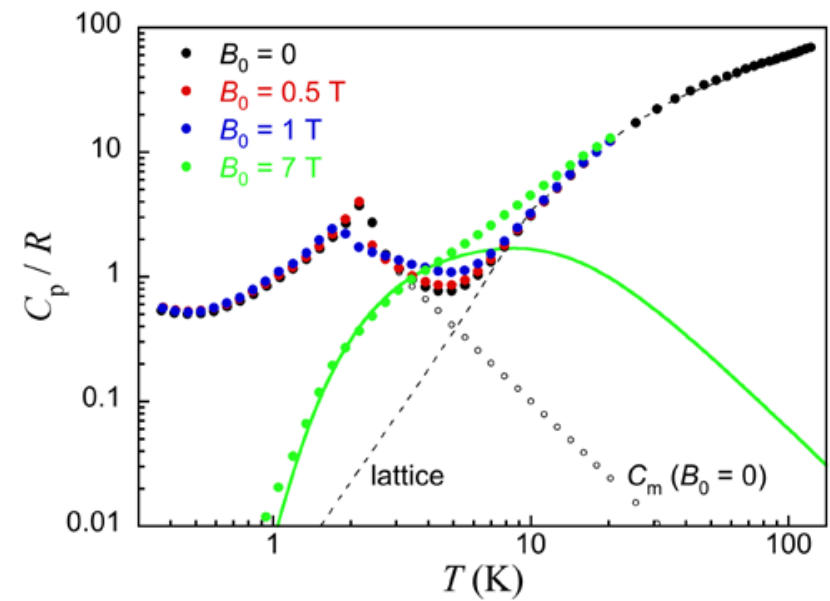

Fig. 4 The molar heat capacity of $\mathbf{1}$, normalized to the gas constant $R$ and collected for the indicated applied fields. Plotted also are: Schottky 25 contribution for $B_{0}=7 \mathrm{~T}$ (solid line), spin-wave linear- $T$ extrapolation and zero-field $C_{\mathrm{m}} \propto T^{-2}$ (both as empty markers), obtained by subtracting the lattice contribution (dashed line) from the total zero-field heat capacity. Inset: Zero-field magnetic entropy of $\mathbf{1}$.

The aforementioned experiments evidence that ferro- and 30 antiferromagnetic interactions are both contributing in the Fe(III) magnetic ordering process. We can push further our analysis by making use of the recent theoretical work by Ito et al. ${ }^{11}$ that sheds light by predicting ferromagnetism for the direct $d$ - $d$ exchange interaction along the same $1 \mathrm{D}$ chain topology as in $\mathbf{1}$. According 35 to their model, we envisage $J / k_{\mathrm{B}}=0.22 \mathrm{~K}$ for such an intrachain coupling. Next, from the value of $2.2 \mathrm{~K}$ as the temperature for the ordering process between the chains, we can estimate the interchain coupling $J$ ' by using the Oguchi's model, ${ }^{19}$ obtaining $\left|J^{\prime}\right| /|J| \approx 0.6$, and therefore $J^{\prime} / k_{\mathrm{B}} \approx-0.13 \mathrm{~K}$ on basis of the above.

40 In summary, we have shown that using simple tetrabromoferrate(III) ions formed in-situ allows the isolation of the first spin crossover (SCO) compound with paramagnetic tetrahaloferrate ions. In this original material, the spin crossover phenomenon of the cationic sublattice coexists with a magnetic
45 order of the anionic sub-lattice. The strategy can certainly be expanded to many spin crossover systems based on a cationic complex, and could thus open a synthetic path to a whole new range of multifunctional spin crossover-based materials.

\section{Notes and references}

$50{ }^{a}$ Instituto de Ciencia de Materiales de Aragón (ICMA), CSIC Universidad de Zaragoza, Departamento de Fisíca de la Materia Condensada, Pl. San Francisco s/n, 50009 Zaragoza, Spain; Fax: 34976 76123; Tel: 34976 762461; E-mail: roubeau@unizar.es

${ }^{b}$ Instituto de Ciencia de Materiales de Aragón (ICMA), CSIC -

55 Universidad de Zaragoza, Sede Campus Río Ebro, María de Luna, 3, 50018 Zaragoza, Spain.

† Electronic Supplementary Information (ESI) available: synthetic details, SEM images of 1, relevant distances/angles for $\mathbf{1}$ and $\mathbf{2}$, additional views of the structures of 1-2, cifs of 1-2. See DOI: 10.1039/b000000x/

$60 \ddagger$ Crystal data for 1: $\left[\mathrm{C}_{12} \mathrm{H}_{24} \mathrm{FeN}_{24}\right]\left(\mathrm{FeBr}_{4}\right)_{2}$. Data were collected at $296 /$ $104 \mathrm{~K}$, trigonal, space group $P-3$ (no. 147) with $a=12.683(2) /$ 12.9487(1) $\AA, c=6.7890(14) / 6.9577(1) \AA, V=947.0(3) / 1010.30(2)$ $\AA^{3}, \mathrm{Z}=1, \rho_{\text {calcd }}=2.299 / 2.155 \mathrm{~g} / \mathrm{cm}^{3}, \mu=10.523 / 9.017 \mathrm{~mm}^{-1} .26453 /$ 16943 reflections were measured, $1342 / 1383$ of which were 65 independent. Refinement converged at final $\mathrm{w} R 2=0.0978 / 0.0578, R 1=$ $0.0359 / 0.0280$ and $S=1.080 / 0.861$ (for $1339 / 903$ reflections with $I>$ $2 \sigma(I))$. Crystal data for 2: $\left[\mathrm{C}_{12} \mathrm{H}_{24} \mathrm{ZnN}_{24}\right]\left(\mathrm{FeBr}_{4}\right)_{2} \cdot 2$. Data were collected at $296 \mathrm{~K}$, trigonal, space group $P-3$ (no. 147) with $a=12.9404$ (4) $\AA$, $c=$ 6.9522(3) $\AA, V=1008.20(6) \AA^{3}, \mathrm{Z}=1, \rho_{\text {calcd }}=2.176 \mathrm{~g} / \mathrm{cm}^{3}, \mu=9.270$ $70 \mathrm{~mm}^{-1} .6659$ reflections were measured, 1382 of which were independent. Refinement converged at final $\mathrm{w} R 2=0.0974, R 1=0.0477$ and $S=1.057$ (for 1060 reflections with $I>2 \sigma(I)$ ).

${ }^{1}$ E. Coronado, J. R. Galán-Mascarós, C. J. Gómez-García and V. Laukhin, Nature, 2000, 408, 447-449; J. S. Miller, Angew. Chem. Int. Ed., 2003, 42, 27-29.

${ }^{2}$ A. B. Gaspar, V. Ksenofontov, M. Seredyuk and P. Gütlich, Coord. Chem. Rev., 2005, 249, 2661-2676 ; A. Bousseksou, G. Molnár, L. Salmon and W. Nicolazzi, Chem. Soc. Rev., 2011, 40, 3313-3335.

${ }^{3}$ P. Gütlich, A. Hauser, H. Spiering, Angew. Chem. Int. Ed., 1994, 33, 2024-2054.

${ }^{4}$ A. B. Gaspar, M. Seredyuk and P. Gütlich, Coord. Chem. Rev., 2009, 253, 2399-2413 ; O. Roubeau, A. Colin, V. Schmitt and R. Clérac, Angew. Chem. Int. Ed., 2004, 43, 3283-3286.

${ }^{5}$ E. Coronado, C. Martí-Gastaldo, E. Navarro-Moratalla, A. Ribera, S. J. Blundell and P. J. Baker, Nature Chem., 2010, 2, 1031-1036; M. Clemente-León, E. Coronado, C. Martí-Gastaldo and F. M. Romero, Chem. Soc. Rev., 2011, 40, 473-497; G. Rogez, C. Massobrio, P. Rabu and M. Drillon, Chem. Soc. Rev., 2011, 40, 1031-1058.

${ }^{6}$ M. Clemente-León, E. Coronado, M. López-Jordà, C. Desplanches, S. 90 Asthana, H. Wang and J-F. Létard, Chem. Sci., 2011, 2, 1121-1127; M. Clemente-León, E. Coronado, M. López-Jordà and J. C. Waerenborgh, Inorg. Chem., 2011, 50, 9122-9130.

${ }^{7}$ J. H. Yoon, D. W. Ryu, S. Y. Chi, H. C. Kim, E. K. Koh, J. Tao and C. S. Hong, Chem. Commun., 2011, 47, 10416-10418.

$9_{95}^{8}$ T. S. Venkatakrishnan, S. Sahoo, N. Bréfuel, C. Duhayon, C. Paulsen, A-L. Barra, S. Ramasesha, J-P. Sutter, J. Am. Chem. Soc., 2010, 132, 6047-6056.

${ }^{9}$ G. Aromí, L. A. Barrios, O. Roubeau and P. Gamez, Coord. Chem. Rev., 2011, 255, 485-546.

$100{ }^{10}$ E. Coronado and P. Day, Chem. Rev., 2004, 104, 5419-5448.

${ }^{11}$ M. Takenaka, T. Kawakami, A. Ito, K. Kinoshita, Y. Kitagawa, S. Yamanaka, K. Yamagushi and M. Okamura, Polyhedron, 2011, 30, 3284-3291.

${ }^{12}$ M. Wang, H. Fujiwara, T. Sugimoto, S. Noguchi and T. Ishida, Inorg. 105 Chem., 2005, 44, 1184-1186.

${ }^{13}$ also with Metz as ligand: L. Wiehl, Acta Cryst., 1993, B49, 289-303.

${ }^{14}$ F. A. Cotton and C. A. Murillo, Inorg. Chem., 1975, 14, 2467-2469.

${ }^{15}$ widely-used in cases where calorimetric data are available, see $\mathrm{M}$. Sorai, Chem. Rev., 2006, 106, 976-1031.

${ }_{110}^{16}$ T. Nakamoto, Z. C. Tan and M. Sorai, Inorg. Chem., 2001, 40, 38053809; O. Roubeau, M. de Vos, A. F. Stassen, R. Burriel, J. G. Haasnoot and J. Reedijk, J. Phys. Chem. Solids, 2003, 64, 1003-1013. 
${ }^{17}$ M. Evangelisti, F. Luis, L. J. de Jongh and M. Affronte, J. Mater. Chem., 2006, 16, 2534-2549.

${ }^{18}$ L. J. de Jongh and A. R. Miedema, Adv. Phys., 2001, 50, 947-1170.

${ }^{19}$ T. Oguchi, Phys. Rev., 1964, 133, A1098-A1099. 